\title{
Betroffenenbeteiligung im Gesetzgebungsverfahren
}

\author{
von \\ Prof. Dr. Matthias Rossi, Universität Augsburg
}

Einleitung

Vor Gericht hat jedermann das Recht, gehört zu werden. Dieser Anspruch auf „rechtliches Gehör“" ist in Art. 103 Abs. 1 GG als grundrechtsgleiches Recht ausgestaltet, so dass eine Verletzung mit der Verfassungsbeschwerde vor dem Bundesverfassungsgericht geltend gemacht werden kann. Auch vor Maßnahmen der Verwaltung muss grundsätzlich eine Anhörung durchgeführt werden. Dieses Recht auf „administratives Gehör" ist für die meisten Fälle einfachgesetzlich normiert, ${ }^{1}$ darüber hinaus aber auch verfassungsrechtlich fundiert. Es wird z. T. aus einer analogen Anwendung des Art. 103 GG, ${ }^{2}$ z.T. aus einem weiten Verständnis des Art. 19 Abs. 4 $\mathrm{GG}^{3}{ }^{3}$ mitunter aus der Menschenwürde, ${ }^{4}$ überwiegend aber aus dem Rechtsstaatsprinzip $^{5}$ bzw. den jeweils betroffenen Grundrechten abgeleitet. ${ }^{6}$ Gegenüber Maßnahmen des Gesetzgebers besteht ein solches Recht dagegen nicht. Der naheliegende Grund für das Fehlen eines solchen Rechts - man mag es als Recht auf „legislatives Gehör" bezeichnen - liegt darin, dass formelle Gesetze regelmäßig abstrakter und genereller Natur sind und deshalb grundsätzlich alle Personen gleichermaßen betreffen.? Sie müssen schon deshalb nicht individuell am Gesetzgebungsverfahren beteiligt werden, weil sie in diesem Verfahren vom Parlament repräsentiert werden, ihre Interessen also von den Abgeordneten als „Vertreter des ganzen Volkes“ wahrgenommen werden.

1 Vgl. bspw. $₫ 28$ Abs. 1 VwVfG; $\$ 91$ AO, $\$ 24$ SGB X.

${ }^{2}$ Rudolf Wassermann, AK-GG, Art. 103, Kn. 23.

${ }^{3}$ Carl Hermann Ule, Zur Bedeutung des Rechtsstaatsbegriffs in der Rechtsprechung des Bundesverwaltungsgerichts, DVBl. 1963, S. 475 (477 f.).

' Friedhelm Hufen, Fehler im Verwaltungsverfahren, 1986, S. 127f.; Ferdinand Otto Kopp. Verfassungsrecht und Verwaltungsverfahrensrecht, 1971, S. $30 \mathrm{ff}$.

${ }^{5}$ Eberhard Schmidt-Aßmann, in: Maunz/Dürig, Grundgesetz Kommentar, 64. EL 2012, Art. 103 Rn. 6.

6 Philip Kunig, Das Rechtsstaatsprinzip, Überlegungen zu seiner Bedeutung für das Verfassungsrecht der Bundesrepublik Deutschland, 1986, S. 374.

${ }^{7}$ Christoph Gusy, Das Grundgesetz als normative Gesetzgebungslehre?, ZRP 1985, S. 291 (296). 
Doch diese theoretische Betrachtung geht in vielen Fällen an der Wirklichkeit vorbei. Rekurriert man weniger auf die rechtlichen als vielmehr auf die tatsächlichen Auswirkungen von Gesetzen, ist nicht zu bestreiten, dass viele, vielleicht gar die meisten Gesetze einzelne Personen oder Personengruppen stärker betreffen als andere: Das Hochschulrecht, das Jagdrecht, das Treibhausgasemissionshandelsrecht seien als einige willkürlich gewählte Beispiele für Gesetze genannt, die wegen ihres Inhalts unmittelbar nicht für jeden, sondern nur für einen bestimmten, relativ eng umgrenzten Personenkreis von Bedeutung sind. Eine solche spezifische Betroffenheit dürfte in der Gesetzgebungspraxis sogar die Regel sein. Dennoch sieht das formelle Gesetzgebungsverfahren keine Beteiligung, kein legislatives Gehör solcher Personen vor, deren Interessen von dem jeweiligen Gesetz in besonderer Weise berührt werden.

Diese Beobachtung ist nicht neu. ${ }^{8}$ Allerdings gab es in jüngerer Zeit verschiedene Impulse, die von einem Gesetz besonders betroffenen Personen auch in besonderer Weise in das Gesetzgebungsverfahren einzubinden. Im Vorfeld und in Vorbereitung eines „Vertrags über eine Verfassung für Europa" formulierte die Europäische Kommission im Jahre 2001 in ihrem Weißbuch „Europäisches Regieren"9 zahlreiche Maßnahmen mit dem Ziel, die Akzeptanz der Europäischen Politik und des Europäischen Rechts durch die Bürger zu stärken. Dabei wurde unter der Maxime „Bessere Einbindung aller Akteure und größere Offenheit“ eine „effektivere und transparentere Konsultation als Herzstück der EU-Politikgestaltung“ bezeichnet. ${ }^{10}$ Auch der sog. Mandelkern-Bericht aus demselben Jahr schlug unter dem Titel „Moderner Staat - Moderne Verwaltung. Auf dem Weg zu besseren Gesetzen“"1l ein grundsätzliches Konsultationsverfahren beim Erlass von Rechtsakten vor. Er versprach sich von dieser Art der Einbeziehung der betroffenen Parteien einen ,inhaltlichen und demokratischen Mehrwert". ${ }^{12}$ Weiterhin wurden auch im Rahmen des Programms der Bundesregierung „Moderner Staat - moderne Verwaltung“ unterschiedliche Beteiligungsmöglichkeiten von Betroffenen am Rechtsetzungsverfahren erörtert, überwiegend unter Rückgriff auf entsprechende Vorarbeiten in den Sozialwissenschaften und in der Rechtswissenschaft. Als Stichworte aus diesen Überlegungen seien nur die kooperative Rechtserzeugung, ${ }^{13}$ die Koregulierung ${ }^{14}$ oder auch das paktierte Gesetz ${ }^{15}$ genannt. Alle diese Entwicklungen wurden von dem Gutachten „Gute Gesetzgebung“ aufgenommen, das Gunnar Folke Schuppert im Jahre 2002 im Auftrag des

\footnotetext{
${ }^{8} \mathrm{Zu}$ dem Befund und möglichen Konsequenzen vgl. bspw. Thomas Roser, Anhörung von Betroffenen im Gesetzgebungsverfahren - Können wir von ausländischen Erfahrungen lernen?, ZParl 15 (1984), S. 534 (536ff.); Raimund Wimmer, Die Wahrung des Grundsatzes des rechtlichen Gehörs, DVBl. 1985, S. 773 (775); Helmuth Schulze-Fielitz, Theorie und Praxis parlamentarischer Gesetzgebung - besonders des 9. Deutschen Bundestages (1980-1983), 1988, S. $510 \mathrm{f}$.

9 KOM (2001) 428.

${ }^{10} \mathrm{KOM}(2001)$ 428, S. 20.

1 Die Expertengruppe unter dem Vorsitz von Dieudonné Mandelkern war von den europäischen Ministern für die öffentliche Verwaltung einberufen worden, um Empfehlungen zur Umsetzung des Lissabon-Prozesses vorzubereiten.

12 Dieudonné Mandelkem, Moderner Staat - Moderne Verwaltung, 2002, S. 38.

13 Gunnar Folke Schuppert, Gute Gesetzgebung, ZG, Sonderheft 2003, S. 75 f.

${ }^{14} \operatorname{KOM}(2001) 428$, S. 13, 40.

$15 \mathrm{Vgl}$. hierzu auch Peter M. Huber, Konsensvereinbarungen und Gesetzgebung, ZG 2002, S. 245 ff.
} 
Bundesministeriums der Justiz erstellt hat. ${ }^{16}$ Schließlich hat sich auch der Deutsche Juristentag im September 2004 im Rahmen des Themas „Wege zu besserer Gesetzgebung" mit der Frage befasst, ob Anhörungen im Rechtsetzungsverfahren intensiviert und verrechtlicht werden sollten. ${ }^{17}$ Und das aktuelle Thema des 69 . Deutschen Juristentags aus dem Jahre 2012 weist mit „Neue Formen der Bürgerbeteiligung? Planung und Zulassung von Projekten in der parlamentarischen Demokratie“ in eine ähnliche Richtung, wenngleich hier wohl eher eine gegenständlich definierte, weniger eine formal begründetc Bürgerbctciligung betrachtet werden soll, so dass im Fokus in erster Linie Verwaltungsverfahren und nur am Rande auch Gesetzgebungsverfahren stehen werden.

Während diese Impulse überwiegend rechtspolitischer Natur sind, ist mit dem Vertrag über die Europäische Union in der Fassung des „Lissabon-Vertrages“ (EUV) ein rechtlicher Grundstein für eine Betroffenenbeteiligung im Gesetzgebungsverfahren gelegt worden. Im Titel II „Bestimmungen über die demokratischen Grundsätze" unterscheidet der Vertrag zwischen dem Grundsatz der repräsentativen Demokratie (Art. 10) und dem Grundsatz der partizipativen Demokratie (Art. 11), wenn diese im Vergleich zum gescheiterten Verfassungsvertrag auch nicht ausdrücklich so benannt wird. Zur partizipativen Demokratie gehört dabei nicht nur die Verpflichtung der Organe der Union, ,einen offenen, transparenten und regelmäßigen Dialog mit den repräsentativen Verbänden und der Zivilgesellschaft“" zu pflegen (Abs. 2). Vielmehr führt die Europäische Kommission nach Art. 11 Abs. 3 EUV „umfangreiche Anhörungen der Betroffenen durch, um die Kohärenz und die Transparenz des Handelns der Union zu gewährleisten." Zwar ist dieses primärrechtliche Anhörungsrecht unabhängig davon, dass es der Konkretisierung ebenso fähig wie bedürftig ist, für die Beteiligung von Betroffenen im nationalen Gesetzgebungsverfahren rechtlich ohne Bedeutung, denn es verpflichtet nur die Europäische Kommission und somit noch nicht einmal die (anderen) Legislativorgane der Europäischen Union. ${ }^{18}$ Gleichwohl spiegelt Art. 11 Abs. 3 EUV in normativer Form eine Entwicklung wider, die auch für die nationale Rechtsetzung die Fragen aufwirft, ob die Beteiligung von Betroffenen am Gesetzgebungsverfahren als Maxime guter Gesetzgebung begriffen werden kann (I) und ob ein derartiges legislatives Gehör rechtlich normiert werden sollte (II). ${ }^{19}$

${ }^{16}$ Gunnar Folke Schuppert, Gute Gesetzgebung, ZG, Sonderheft 2003.

$17 \mathrm{~S}$. insb. Peter Blum, Wege zu besserer Gesetzgebung - sachverständige Beratung, Begründung, Folgeabschätzung und Wirkungskontrolle, Gutachten I zum 65. DJT, 2004, S. I 121 u. passim.

$18 \mathrm{Vgl}$. Matthias Rossi, Interparlamentarische Demokratie? - Zur Einbindung der nationalen Parlamente in die Rechtsetzung der Europäischen Union, in: Michael Kloepfer (Hrsg.)., Gesetzgebung als wissenschaftliche Herausforderung, Gedächtnisschrift für Thilo Brandner 2011, S. 47 (49).

19 Bezugsgegenstand der nachfolgenden Überlegungen ist in erster Linie das innerstaatliche parlamentarische Gesetzgebungsverfahren auf Bundes- wie auf Landesebene. Die Rechtsetzung in der Europäischen Union wird dagegen nur am Rande tangiert, die Rechtsetzung in Internationalen Organisationen bleibt außer Betracht. 


\section{Legislatives Gehör als Maxime guter Gesetzgebung}

Denn in der Tat kann eine Betroffenenbeteiligung im Gesetzgebungsverfahren durchaus als Maxime guter Gesetzgebung begriffen werden, wenn man sie weniger als Ausdruck von Lobbyismus denn als Zeichen von Partizipation begreift (1.) und ihre Funktionen stärker betont (2.) als ihre Risiken (3.).

\section{Lobbyismus oder Partizipation?}

In Deutschland firmiert die Einbringung privater Interessen in das formelle Gesetzgebungsverfahren vor allem unter dem eher negativ konnotierten Begriff des Lobbyismus, ${ }^{20}$ der sich jedenfalls nicht ohne weiteres als Maxime guter Gesetzgebung verstehen lässt. In dem Begriff schwingt etwas Klandestines, etwas Anrüchiges, ja zuweilen etwas Verbotenes mit. Und auch der zunächst positiv, jedenfalls aber neutral verwendete Begriff der „Beratung“ steht nach den zumindest in ihrem Ausmaße umstrittenen Dienstleistungen, die insbesondere die Bundesregierung von Unternehmensberatern und Rechtsanwälten in Anspruch genommen hat, ${ }^{21}$ wenn vielleicht auch zu Unrecht als Synonym für eine illegitime Beeinflussung politischer Entscheidungen. ${ }^{22}$

Warum der in anderen Ländern durchaus positiv verwendete Begriff des Lobbyismus in Deutschland immer noch so viel pejorative Assoziationen weckt, liegt womöglich an der traditionellen deutschen Staatszentriertheit, an der Vorstellung, der Staat allein sei Garant des Gemeinwohls. ${ }^{23}$ Die Pluralismustheorien haben dagegen die Sicherung und Gewährleistung des Gemeinwohls von jeher dem Wechselspiel der verschiedenen gesellschaftlichen Gruppen überlassen. ${ }^{24}$ Unabhängig von solchen politikwissenschaftlichen Überlegungen ist jedenfalls zu beobachten, dass der Anstoß zu einer verstärkten Betroffenenbeteiligung im Gesetzgebungsverfahren mit einer weitreichenden Privatisierung und Liberalisierung von Staatsaufgaben sowie einer Aktivierung der Bürgergesellschaft einhergeht und dass alle diese Entwicklungen ihren Ursprung in der Europäischen Union haben. ${ }^{25}$

20 Frank Daumann, Interessenverbände im politischen Prozess. Eine Analyse auf Grundlage der Neuen Politischen Ökonomie, 1999, S. 10.

${ }^{21}$ Nachzulesen etwa in der Zeit v. 5.2. 2004, S. 9 ff.; s. auch Antwort der Bundesregierung auf die Kleine Anfrage mehrerer Bundestagsabgeordneter, BT-Drs. 15/2458; wissenschaftlich aufbereitet in den verschiedenen Beiträgen in: Michael Kloepfer (Hrsg.), Gesetzgebungsoutsourcing - Gesetzgebung durch Rechtsanwälte?, 2011, passim.

22 Vgl. die einzelnen Beiträge in Svenja Falk et al. (Hrsg.), Handbuch Politikberatung,

${ }^{23} \mathrm{Vgl}$. Ulrich von Alemann, Lobbyismus heute, Neue Herausforderungen durch Globalisierung, Europäisierung und Berlinisierung, 2000, S. 2.

${ }^{24}$ Grundlegend Emst Frankel, Reformismus und Pluralismus, 1973, passim. Zur Adaption auf die Gegenwart vgl. Oliver Mross, Bürgerbeteiligung am Rechtsetzungsprozess in der Europäischen Union. Ein Beitrag zur Stärkung der demokratischen Legitimation?, 2010, S. 239; zu den Strukturschwächen des Pluralismus schon Helmurh Schulze-Fielitz, Theorie und Praxis parlamentarischer Gesetzgebung besonders des 9. Deutschen Bundestages (1980-1983), 1988, S. $393 \mathrm{ff}$.

25 Ähnlich Martin Nettesheim, in: Grabitz/Hilf (Hrsg.), Das Recht der Europäischen Union, 46. EL 2011, Art. 11 EUV Rn. 6. 
Möglicherweise ist in Bezug auf den Lobbyismus tatsächlich ein Bewertungswandel angezeigt. Zwar werden die Grenzen zwischen legitimer Wahrnehmung und Durchsetzung eigener Interessen auf der einen Seite und illegitimer Einflussnahme auf den politischen Entscheidungsprozess auf der anderen Seite immer changierend bleiben. Doch die neuen politischen Ansätze weisen in gewissem Maße auf positive Aspekte der Einbeziehung von privaten Akteuren in den Gesetzgebungsprozess hin. $\mathrm{Zu}$ einseitig wird bislang der Lobbyismus in Deutschland allein aus der Perspektive des einflussnehmenden Privaten beäugt ${ }^{26}$ zu wenig wird berücksichtigt, welche Vorteile sich aus einer Betroffenenbeteiligung für den Prozess der Gesetzgebung, damit zugleich für den Inhalt des Gesetzes und somit letztlich für das Gemeinwohl ergeben können.

\section{Funktionen einer Betroffenenbeteiligung}

Von den zahlreichen Funktionen, die einer Betroffenenbeteiligung (je nach wissenschaftlicher Perspektive) zugeschrieben werden können, ${ }^{27}$ sollen hier nur die folgenden hervorgehoben werden:

\section{a) Wechselseitige Informationsfunktion}

Einer Betroffenenbeteiligung kommt vor allem eine wechselseitige Informationsfunktion zu. ${ }^{28}$ Auf der einen Seite kann die Anhörung von Betroffenen den Gesetzgeber über den zu regelnden Gegenstand und die davon berührten Interessen näher informieren. Diese Informationen sind unerlässlich für die Beantwortung der Fragen, ob überhaupt ein Regelungsbedarf besteht, welche Rechtsgüter möglicherweise betroffen sind, welche Konfliktlagen und Überschneidungsbereiche zu berücksichtigen sind, ob und welche Regelungsalternativen zur Verfügung stehen und welche Folgen sich aus dem Gesetz ergeben würden - Fragen, zu denen die Begründung eines Gesetzentwurfs nach $\$ 43$ GGO explizit Stellung nehmen soll, Fragen auch, die der Gesetzgeber beantworten muss, um seinen umfassenden Aufklärungspflichten nachzukommen. ${ }^{29}$ Zwar verfügen die Gesetzgebungsorgane, verfügt insbesondere die Ministerialverwaltung regelmäßig über entsprechende Kenntnisse, doch kann das Informationsspektrum durch die Anhörung der Betroffenen erweitert und eine

${ }^{26} \mathrm{Vgl}$. hierzu z. B. Thomas Hoeren, Was Däubler-Gmelin und Hunzinger gemeinsam haben - Die zehn Verfahrensgebote der Informationsgerechtigkeit, NJW 2002, S. 3303.

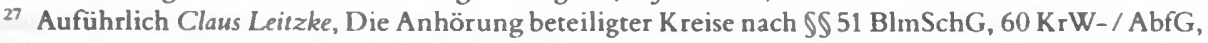
17 Abs. 7 ChemG, 6 WRMG, 20 BBodSchG. Funktionen, Merkmale, Verbesserungsmöglichkeiten, 1999, S. $41 \mathrm{ff}$.

28 Florian Becker, Kooperative und konsensuale Strukturen in der Normsetzung, 2005, S. 113.

29 Deutlich BVerfGE 50, 50 (51): „Das Verfassungsgericht hat insbesondere nachzuprüfen, ob der Gesetzgeber den für seine Maßnahmen erheblichen Sachverhalt zutreffend und vollständig ermittelt und dem Gesetz zugrunde gelegt hat, ob er alle Gemeinwohlgründe sowie die Vorteile und Nachteile der gesetzlichen Regelung umfassend und in nachvollziehbarer Weise abgewogen hat und ob der gesetzgeberische Eingriff geeignet, erforderlich und verhältnismäßig ist und die Gebote der Sachgerechtigkeit und Systemgerechtigkeit beachtet.“ 
Ausgewogenheit der Informationsgrundlage sichergestellt werden. ${ }^{30}$ Von besonderer Bedeutung ist die zusätzliche Informationsbasis vor allem, wenn die Gesetzgebungsorgane selbst nicht über den notwendigen Sachverstand hinsichtlich der zu regelnden Materie verfügen. In solchen Fällen wird eine vernünftige Normsetzung ohne das Fachwissen der Betroffenen gar nicht möglich sein. ${ }^{31}$ So erscheint es etwa durchaus angezeigt, bei einer Neuordnung des Chemikalienrechts den Verband der Chemischen Industrie um eine Stellungnahme zu bitten und diese Stellungnahme im weiteren Rechtsetzungsverfahren auch zu berücksichtigen.

Auf der anderen Seite erhalten auch die angehörten Betroffenen nähere Informationen über die Pläne des Gesetzgebers. Sie können sich frühzeitig auf neue rechtliche Regelungen einstellen und entsprechend disponieren, müssen dies angesichts der Vorwirkungen von Gesetzen gegebenenfalls sogar. ${ }^{32}$ Während also im Vordergrund des Informationsflusses von den Betroffenen an die Gesetzgebungsorgane tatsächliche Informationen stehen, betrifft die Informationsvermittlung von den gesetzgebenden Organen an die Betroffenen in erster Linie rechtliche Informationen.

\section{b) Qualitätssicherung}

Die breitere und tiefere Informationsgrundlage der Gesetzgebungsorgane kann grundsätzlich die inhaltliche Qualität der Gesetze verbessern. Denn wenn die Verfassung das Parlament bzw. seine Mitglieder auch durch die Unmittelbarkeit der Wahl und die anderen Wahlrechtsgrundsätze in besonderer Weise legitimiert, stellt sie an deren Qualifikation doch keine Anforderungen. ${ }^{33}$ Erst eine umfassende Informationsbasis bietet deshalb die Gewähr für die sachliche Richtigkeit von Gesetzen. ${ }^{34}$ Das bedeutet nicht, dass die unzureichende Berücksichtigung relevanter Informationen - ähnlich wie bei der Ausübung von Ermessen oder planerischer Abwägung durch die Exekutive - zu einer sachlich unrichtigen oder gar rechtswidrigen Entscheidung des Gesetzgebers führen muss. Dem Gesetzgeber ist vielmehr eine legislative Einschätzungsprärogative zuzuerkennen, die es ihm insbesondere unter Zeitdruck auch erlaubt, auf ungesicherter tatsächlicher Grundlage zu entscheiden. ${ }^{35}$ Er hat seine Entscheidungen insofern nicht auf vollkommener, sondern auf optimaler Informationsgrundlage zu treffen. ${ }^{36}$ Dabei ist allerdings in der Regel erstens davon auszugehen, dass eine optimale Informationsgrundlage nur durch die Einbeziehung

30 Christoph Gusy, Das Grundgesetz als normative Gesetzgebungslehre?, ZRP 1985, S. 291 (292); zum „parzellierenden Wissen“ der Gesetzgebungsorgane Gregor Kirchhof, Die Allgemeinheit des Gesetzes, 2009, S. $50 \mathrm{ff}$.

31 Axel Burghart, Die Pflicht zum guten Gesetz, 1996, S. 125 mit Hinweis auf weitere Erkenntnisquellen.

32 Hierzu grundlegend Michael Kloepfer, Vorwirkung von Gesetzen, 1974, passin.

${ }_{33}$ Christoph Gusy, Das Grundgesetz als normative Gesetzgebungslehre?, ZRP 1985, S. 291 (297).

34 Alexander Ruch, Sachkunde und Entscheidungskompetenz in der Rechtsetzung, in: Kurt Eichenberger u.a. (Hrsg.): Grundfragen der Rechtsetzung, Basel 1978, S. 205 (208); Helmuth Schulze-Fielitz, Wege, Umwege oder Holzwege zur besseren Gesetzgebung durch sachverständige Beratung, Begründung, Folgeabschätzung und Wirkungskontrolle?, JZ 2004, S. 862 (868).

${ }^{35}$ Ausführlich hierzu Klaus Meßerschmid, Gesetzgebungsermessen, 2000, S. 954 m.w. N.

36 Klaus Meßerschmidt, Gesetzgebungsermessen, 2000, S. 955. 
der von dem Gesetz in besonderer Weise betroffenen Personen erlangt werden kann. Und zweitens kann grundsätzlich auch angenommen werden, dass die Berücksichtigung dieser Informationen grundsätzlich die sachliche Richtigkeit des Gesetzes verbessert. $^{37}$

\section{c) Akzeptanzsicherung}

Neben der Qualität der Normen kann die Beteiligung von Betroffenen im Gesetzgebungsverfahren auch die Akzeptanz der erlassenen Regeln verbessern. ${ }^{38}$ Dies ist ausweislich des Weißbuchs der Kommission und auch des Mandelkern-Berichts sogar die Hauptfunktion der Betroffenenbeteiligung. Sie gehen davon aus, dass Normen von ihren Adressaten eher befolgt werden, wenn sie an deren Erlass beteiligt waren. Besonders akzeptanzsteigernd wirke es dabei, wenn die Betroffenen nicht nur angehört, sondern ihre Anstöße auch in dem jeweiligen Gesetz berücksichtigt wurden. ${ }^{39}$ In solchen Fällen wird die Akzeptanzfunktion unterstützt durch eine Identifizierung der privaten Akteure mit dem Gesetzgeber. Sie erscheinen (sich selbst) nicht mehr nur als obrigkeitsstaatsunterworfene Bürger, sondern als aktive Gestalter ihrer eigenen Belange und zugleich der des Gemeinwohls.

\section{d) Vollzugssicherung}

Geht man weiter davon aus, dass alle von einer Norm in besonderer Weise Betroffenen auch im Rahmen des Gesetzgebungsverfahrens beteiligt wurden, sie also die Gelegenheit hatten, ihre Interessen zu äußern, geht man weiterhin davon aus, dass der Gesetzgeber die dieserart artikulierten Interessen im weiteren Gesetzgebungsverfahren auch berücksichtigt hat, dann folgt aus der (vermuteten) Akzeptanz der dieserart entstanden Norm auch eine hohe Wahrscheinlichkeit ihrer Beachtung. Insofern dient die Betroffenenbeteiligung möglicherweise auch der Vollzugssicherung ${ }^{40}$. Denn die Gesetze werden in diesen Fällen nicht nur wegen des ihnen eigenen, auf die parlamentarische Legitimation zurückgehenden (formalen) Rechtsan-

37 Christian von Hammerstein, Verbesserung der Gesetzgebung durch neue Instrumente und Methoden, in: Hermann Hill (Hrsg.), Zustand und Perspektiven der Gesetzgebung, Vorträge und Diskussionsbeiträge der 56. Staatswissenschaftlichen Fortbildungstagung 1988 der Hochschule für Verwaltungswissenschaften Speyer, 1989, S. 141 (142f.); in diesem Sinne auch Julian Krüper, Lawfirm - legibus solutus? Legitimität und Rationalität des inneren Gesetzgebungsverfahrens und das „Outsourcing“ von Gesetzentwürfen, JZ 2010, S. 655 (660).

${ }^{38}$ Fritz Ossenbühl, in: Josef Isensee/Paul Kirchhof (Hrsg.), HdBStR Bd. 3, 1. Auf. 1988, $₫ 63$ Rn. 11.

${ }^{39}$ Dieudonné Mandelkern, Moderner Staat - Moderne Verwaltung, 2002, S. 37; KOM (2001) 428, S. $13 \mathrm{f}$.

40 Hans-Joachim Mengel, Gesetzgebung und Verfahren, Ein Beitrag zur Empirie und Theorie des Gesetzgebungsprozesses im föderalen Verfassungsstaat, 1997, S. 282; Jürgen Rödig, Zum Stellenwert der Gesetzgebungstheorie in der herkömmlichen Dogmatik sowie der traditionellen Methodenlehre, in: ders. / Eberhard Baden / Harald Kindermann (Hrsg.), Vorstudien zu einer Theorie der Gesetzgebung, 1975, S. 27 (29). 
wendungsbefehls beachtet, sondern auch wegen der (inhaltlichen) Akzeptanz der Norm. ${ }^{41}$

\section{Risiken einer Betroffenenbeteiligung}

Mögen die skizzierten Funktionen und (erhofften) Wirkungen die Betroffenenbeteiligung im Gesetzgebungsverfahren auch als Maxime guter Gesetzgebung erscheinen lassen, so sehr sind doch auch die Risiken, die Nebenwirkungen, ${ }^{42}$ hervorzuheben, die von ihr ausgehen können.

\section{a) Einflussmöglichkeiten von Partikularinteressen}

Zum einen gehen mit der Beteiligung, selbst wenn sie nur als Anhörung ausgestaltet ist, erhebliche Einflussmöglichkeiten von Partikularinteressen einher. Denn die Betroffenen werden einseitig ihre Interessen darstellen, so dass nicht zwingend gewährleistet ist, dass etwaige widerstreitende Interessen ebenfalls in das Gesetzgebungsverfahren eingebracht werden. ${ }^{43}$ Doch muss dies einer Einbindung von Betroffenen in das Gesetzgebungsverfahren nicht entgegenstehen. Denn ungeachtet der zahlreichen theoretischen und praktischen Untersuchungen zu der Frage, die im Zusammenhang mit dem Übergang von der Erfüllungs- zur Gewährleistungsverantwortung des Staates immer wieder neu gestellt wurde, ${ }^{44}$ zu der Frage, wie das Gemeinwohl am besten definiert und verwirklicht werden kann, ${ }^{45}$ hängt die Grenze zwischen einer mit den Grundsätzen repräsentativer Demokratie nicht vereinbaren Dominanz von Partikularinteressen und einer dem parlamentarischen Verantwortungsadressaten - das ,ganze Volk“ - entsprechenden Möglichkeit der Stellungnahme doch einerseits von der abstrakten Ausgestaltung des Verfahrens der Betroffenenbeteiligung und andererseits von der Frage ab, wen das gesetzgebende Organ im konkreten Fall als Betroffenen hinzuzieht.

\section{b) Chancengleichheit}

Unmittelbar im Zusammenhang mit dem Risiko einer Dominanz von Partikularinteressen steht die Gefahr, dass Gleichheitsrechte verletzt werden. Denn eine Betroffenenbeteiligung im Gesetzgebungsverfahren steht und fällt, dies wird noch problematisiert werden, mit der Definition der besonderen Betroffenheit und der Aus-

4. Georg Müller, Elemente einer Rechtssetzungslehre, Zürich 2. Aufl. 2006, Rn. 21.

42 Die Unterscheidung in $\$ 44$ GGO zwischen beabsichtigten Wirkungen und unbeabsichtigten Nebenwirkungen unter dem Oberbegriff der Auswirkungen ist wohl das größte Eingeständnis der begrenzten Steuerungskraft von Gesetzen.

${ }^{43}$ Thomas Hoeren, Was Däubler-Gmelin und Hunzinger gemeinsam haben - Die zehn Verfahrensgebote der Informationsgerechtigkeit, NJW 2002, S. 3303.

44 Resümierend Claudio Franzius, Der Gewährleistungsstaat, VerwArch 99 (2008), S. 351 ff.

45 Lesenswert Michael Anderheiden, Gemeinwohl in Republik und Union, 2006, passim. 
wahl der Betroffenen. Die allgemeine Willkürgrenze, die sich aus Art. 3 I GG ebenso wie aus dem Rechtsstaatsprinzip, darüber hinaus möglicherweise auch aus der Gemeinwohlorientierung des Gesetzgebers ableiten lässt, ${ }^{46}$ ist hier ebenso strikt zu beachten wie in Gefahr, missachtet zu werden. Doch auch dieses Risiko lässt sich möglicherweise durch eine Struktur und ein System der Betroffenenbeteiligung minimieren, die gewährleisten, dass alle relevanten Akteure in angemessener und gebührender Weise konsultiert werden.

\section{c) Charakter der repräsentativen Demokratie}

Vor allen Dingen aber darf ein eher theoretisches Problem nicht übersehen werden. In dem $\mathrm{Maße}$, in dem die Betroffenenbeteiligung zu einer politischen Willensbildung außerhalb des Parlaments beiträgt, geht auch der repräsentative Charakter der grundgesetzlichen Demokratie verloren. Schon jetzt ist es häufig zu beobachten, dass eine Unterscheidung zwischen dem von der Regierung formulierten politischen Willen und einem vom Bundestag gefassten Gesetzesbeschluss kaum noch gemacht wird. Zwar bleibt der Gesetzesbeschluss des Bundestages conditio sine qua non für die Wirksamkeit von Recht, aber diese herausgehobene Stellung läuft ins Leere, wenn im Parlament nur die von der Regierung und den Betroffenen erarbeiteten Entwürfe ratifiziert werden. Das Parlament nimmt in solchen Fällen dann nur noch die Funktion eines Hausnotars der Regierung war. Mit der fehlenden materiellen Auseinandersetzung im Parlament geht aber nicht nur der Bedeutungsverlust dieser Institution einher, ausgeschlossen wird vielmehr auch die Öffentlichkeit mit ihrer kontrollierenden Funktion. ${ }^{47}$

Diese seit langem beobachteten Phänomene haben ihre Ursache nicht allein in der Möglichkeit der Betroffenenbeteiligung im inneren Gesetzgebungsverfahren, das sich ja vorrangig in der Entwurfsphase und damit faktisch überwiegend auf Regierungsebene abspielt, aber sie verstärken sich dadurch. ${ }^{48}$

Beide Zielsetzungen, die Betroffenenbeteiligung einerseits und die Wahrung der materiellen parlamentarischen Entscheidungsbefugnis andererseits, lassen sich allerdings in einen Einklang bringen. So könnten Betroffenenbeteiligungen grundsätzlich erst oder jedenfalls auch vor den Ausschüssen des Bundestages durchgeführt werden. Ausreichend wäre es unter Umständen aber auch schon, die im Ministerialbereich durchgeführten Betroffenenbeteiligungen öffentlich auszugestalten oder jedenfalls zu protokollieren.

\footnotetext{
46 Hans-Joachim Mengel, Gesetzgebung und Verfahren, Ein Beitrag zur Empirie und Theorie des Gesetzgebungsprozesses im föderalen Verfassungsstaat, 1997, S. 282 f.

47 Vgl. hierzu Gunnar Folke Schuppert, Gute Gesetzgebung. ZG, Sonderheft 2003, S. 18.

48 Helge Ehler, 2000 Lobbyisten, 613 Abgeordnete, zwei Parteien und eine Regierung, Praktisch kein Gesetz ohne direkte oder indirekte Mitwirkung - Demokratische Legitimierung fehlt, VW 2006, S. 806 .
} 


\section{d) Vertrauensverlust}

Schwerer wiegt hingegen, dass jedenfalls die extensive Praxis einer Betroffenenbeteiligung zu einem Vertrauensverlust in das Recht führen kann. Diese These klingt angesichts der möglichen Akzeptanzsteigerung des mit einer Betroffenenbeteiligung gesetzten Rechts zunächst widersprüchlich. Doch erstens muss hervorgehoben werden, dass die privilegierte Betroffenenbeteiligung nur bei denjenigen zu einer gesteigerten Akzeptanz und Vollzugsbereitschaft führt, die tatsächlich am Rechtsetzungsverfahren beteiligt waren, hingegen zu einer entsprechenden Verringerung bei denjenigen, die trotz einer subjektiv empfundenen Betroffenheit außen vor geblieben sind. Und zweitens sinkt unter Umständen die Bereitschaft, solches Recht zu befolgen, daß ohne jede Beteiligung ${ }^{49}$, also im klassischen Sinne erlassen wird, weil es eben ausnahmsweise alle Bürger gleich und niemanden in besonderer Weise betrifft.

\section{e) Kompatibilität mit anderen Zielen guter Gesetzgebung}

Schließlich muss auch die Kompatibilität einer Betroffenenbeteiligung mit anderen Maximen guter Gesetzgebung hinterfragt werden. In der bis in die Antike zurückreichenden Geschichte guter Gesetzgebungslehren sind zahlreiche Maximen entwickelt worden, die sich auf den Inhalt der zu erlassenden Gesetze beziehen. ${ }^{30}$ Solche inhaltlichen Vorgaben können hier nicht näher betrachtet werden. Stattdessen soll die Vereinbarkeit der Betroffenenbeteiligung mit zwei strukturellen Anforderungen an gute Gesetzgebung thematisiert werden, mit der Forderung nach Transparenz sowie nach Verständlichkeit.

Insoweit ist zunächst zu erinnern, dass die Transparenz des Gesetzgebungsverfahrens vor allem durch die Öffentlichkeit der Plenardebatten im Bundestag (Art. 42 Abs. 1 S. 1 GG) und im Bundesrat (Art. 52 Abs. 3 GG) gewährleistet wird. Die Ausschusssitzungen wie darüber hinaus vor allem auch das ministerielle Verfahren der Entwurfsaufstellung finden dagegen nach geltenden Rechtsvorschriften grundsätzlich nicht öffentlich statt. Wenn eine Betroffenenbeteiligung aber mehr ist bzw. sein soll als eine bloße Anhörung, wenn sie sich zu einem Instrument materieller Entscheidungsfindung herauskristallisiert oder herauskristallisieren soll, dann muss sie grundsätzlich öffentlich stattfinden, um nicht mehr zu verschleiern als zu erhellen. ${ }^{5:}$ Die Ziele der Betroffenenbeteiligung einerseits und der Transparenz andererseits sind

49 Hans Joachim Mengel, Gesetzgebung und Verfahren, Ein Beitrag zur Empirie und Theorie des Gesetzgebungsprozesses im föderalen Verfassungsstaat, 1997, S. 282; Jürgen Rödig, Zum Stellenwert der Gesetzgebungstheorie in der herkömmlichen Dogmatik sowie der traditionellen Methodenlehre, in: ders. / Eberhard Baden/Harald Kindermann (Hrsg.), Vorstudien zu einer Theorie der Gesetzgebung. 1975, S. 27 (29).

50 S. knapp Erk Volkmar Heyen, Historische und philosophische Grundfragen der Gesetzgebungslehre, in: Waldemar Schreckenberger (Hrsg.), Gesetzgebungslehre, 1986, S. 11 ff.; zur Gerechtigkeit als materiellem Ziel der Gesetzgebung Wolfgang Waldstein/Johannes Michael Rainer, Römische Rechtsgeschichte, 10. Auf. 2005, \$9 Rn. 3; zur Entwicklung im 19. Jahrhundert vgl. Sigrid Emmenberger, Gesetzgebungskunst, 2006, S. $29 \mathrm{ff}$.

51 Hans-Joachim Mengel, Grundvoraussetzungen demokratischer Gesetzgebung, Zur Notwendigkeit einer Prozeßordnung des inneren Gesetzgebungsverfahrens, ZRP 1984, S. 153 (156). 
dabei aber wohl schon ausreichend in Einklang gebracht, wenn die Durchführung der Betroffenenbeteiligung protokolliert und publiziert wird, so dass sie rekapituliert werden kann.

Was sodann die Verständlichkeit von Rechtsvorschriften betrifft, so ist diese nicht nur rechtsstaatlich gefordert, ${ }^{52}$ sondern auch zentrale Voraussetzung für eine größere Akzeptanz und bessere Befolgung von Rechtsnormen, wie der Mandelkern-Bericht hervorhebt. Er betont zugleich, dass verständliche Rechtsvorschriften auch aus der Perspektive des Rechtsschutzes geboten sind, weil weniger privilegierte und bildungsfernere Bevölkerungsschichten Schwierigkeiten hätten, ihre Rechte zu verstehen. ${ }^{53}$ Angesichts des Umstands, dass eine Ursache für unverständliche Regelungen im komplizierten Gesetzgebungsverfahren mit seinen zahlreichen Akteuren liegt, ist nicht unbedingt zu erwarten, dass eine zunehmende Betroffenenbeteiligung zu einer besseren Verständlichkeit führt. Freilich wird man hier wohl im Einzelfall differenzieren müssen und grundsätzlich die These wagen können, dass eine Betroffenenbeteiligung im Gesetzgebungsverfahren zwar nicht die Verständlichkeit der Norm in einem absoluten Sinne verbessern wird, aber doch gerade zu einer besseren Verständlichkeit für die Normunterworfen, für die Betroffenen also, führen kann. ${ }^{54}$

\section{Zwischenergebnis}

Um zu einer Antwort auf die erste Frage zu kommen: Die Beteiligung von Betroffenen im Gesetzgebungsverfahren lässt sich mit guten Gründen als eine Maxime guter Gesetzgebung begreifen. Die enge Kommunikation zwischen den Gesetzgebungsorganen und den Betroffenen kann sicherlich zur Verbesserung des Gesetzesinhalts im Sinne seiner sachlichen Richtigkeit beitragen. Allerdings dürfen die Risiken, die von einer Betroffenenbeteiligung ausgehen, nicht ignoriert werden. Sie können allerdings unter Umständen durch eine rechtliche Normierung der Betroffenenbeteiligung minimiert werden.

\section{Rechtliche Normierung der Betroffenenbeteiligung}

Damit sei die zweite Frage aufgeworfen, ob und gegebenenfalls wie eine Beteiligung von Betroffenen am Gesetzgebungsverfahren normiert werden sollte. Die Beantwortung der Frage geht von dem Befund aus, dass eine Betroffenenbeteiligung derzeit nicht verfassungsrechtlich (1.), sondern nur partiell in den Geschäftsordnungen verankert ist (2.). Vor diesem Hintergrund muss die Frage nach der Zulässigkeit eines Gesetzgebungsverfahrensrechts gestellt werden (3.), bevor die Ausgestaltung einer Normierung thematisiert wird (4.).

52 Statt vieler Hellmuth Schulze-Fielitz, in: Horst Dreier (Hrsg.), GG-Kommentar, Bd. 2, 2. Aufl. 2006, Art. 20 (Rechtsstaat) Rn. 141 mit zahlreichen Nachweisen aus Rechtsprechung und Literatur.

53 Dieudonné Mandelkern, Moderner Staat - Moderne Verwaltung, 2002, S. 57.

${ }^{54}$ Axel Burghart, Die Pflicht zum guten Gesetz, 1996, S. 125. 


\section{Keine Verankerung im $G G$}

Im Unterschied zur erwähnten Vorschrift des Art. 11 Abs. 3 EUV schweigt das Grundgesetz nicht nur zu etwaigen Rechten von Betroffenen im Rechtsetzungsverfahren, es verhält sich vielmehr gar nicht zu dem sogenannten inneren Gesetzgebungsverfahren, ${ }^{55}$ demjenigen Verfahren, in dem die Gesetze in Umsetzung politischer Vorgaben vorbereitet und ausgearbeitet werden. Die verfassungsrechtlichen Vorgaben für das Gesetzgebungsverfahren regeln nur das sog. äußere Gesetzgebungsverfahren und setzen dabei mit der Einbringung eines Gesetzesentwurf in den Bundestag bzw. in den Bundesrat relativ spät an.

Demgegenüber kennt bspw. die Bundesverfassung der Schweiz seit der Verfassungsreform von 1999 das sog. „Vernehmlassungsverfahren“, in dem „die Kantone, die politischen Parteien und die interessierten Kreise bei der Vorbereitung wichtiger Erlasse und anderer Vorhaben von großer Tragweite sowie bei wichtigen völkerrechtlichen Verträgen schon im Rahmen des inneren Gesetzgebungsverfahrens zur Stellungnahme eingeladen werden. “56 Ungeachtet seiner verfassungsrechtlichen Normierung auf Bundesebene hat das Vernehmlassungsverfahren eine lange, bis in das 19. Jahrhundert zurückreichende Tradition ${ }^{57}$ und ist auch in den meisten Kantonen vorgesehen. ${ }^{58}$ Dabei darf freilich nicht übersehen werden, dass das Vernehmlassungsverfahren in vielen Fällen in einem direkten Zusammenhang mit den direktdemokratischen Instrumenten steht und bspw. in den Deutschschweizer Kantonen vor allem darauf ausgerichtet ist, die referendumsfähigen Organisationen einzubinden. Nur zum Teil zielt es dagegen auf eine generelle öffentliche Diskussion, ${ }^{59}$ so dass gegenüber einer vorschnellen und undifferenzierten Übertragung auf die deutsche Rechtsordnung und Rechtskultur Vorsicht geboten ist.

\section{Teilweise Verankerung in den Geschäftsordnungen}

Im geltenden nationalen Recht finden sich Vorgaben über die Anhörung von Betroffenen dagegen nur in den Geschäftsordnungen der Gesetzgebungsorgane. Für das äußere Gesetzgebungsverfahren gestattet $\ 70$ GO-BT den Bundestagsausschüssen,

${ }_{55}$ Vgl. hierzu Gunther Schwerdtfeger, Optimale Methodik der Gesetzgebung als Verfassungspflicht, in: Rolf Stödter/Werner Thieme (Hrsg.), Hamburg, Deutschland, Europa. Beiträge zum deutschen und europäischen Verfassungs-, Verwaltungs- und Wirtschaftsrecht, Festschrift für Hans Peter Ipsen zum 70. Geburtstag, 1977, S. 173 ff.; Sven Hölscheidt/ Steffi Menzenbach, Das Gesetz ist das Ziel: Zum Zusammenhang zwischen gutem Verfahren und gutem Gesetz, DÖV 2008, S. 139 ff.

${ }^{56}$ Art. 147 Bundesverfassung Schweiz, s. hierzu Thomas Sägesser, Das Vernehmlassungsverfahren im Schweizerischen Bundesstaat, ZG 2004, S. 364 ff.; sowie ausführlich ders., Vernehmlassungsgesetz, Bundesgesetz vom 18. März 2005 über das Vernehmlassungsverfahren, 2006, passim; zur möglichen Vorbildwirkung Thomas Roser, Anhörung von Betroffenen im Gesetzgebungsverfahren - Können wir von ausländischen Erfahrungen lernen?, ZParl 15 (1984), S. 534 (536ff.).

57 Josef Blaser, Das Vernehmlassungsverfahren in der Schweiz, 2003, S. $23 \mathrm{ff}$.

${ }_{58} \mathrm{Vgl}$. Kurt Nuspliger/Stephan Brunner, Das Vernehmlassungsverfahren in den Deutschschweizer Kantonen, LEGES 2011, S. $239 \mathrm{ff}$.

39 So das Resümee von Kurt Nuspliger/Stephan Brunner, Das Vernehmlassungsverfahren in den Deutschschweizer Kantonen, LEGES 2011, S. 239 (245). 
„zur Informationen über einen Gegenstand seiner Beratung ... öffentliche Anhörungen von Sachverständigen, Interessenvertretern und anderen Auskunftspersonen vorzunehmen." Von größerer praktischer Bedeutung sind jedoch die Anhörungsmöglichkeiten im vorbereitenden inneren Gesetzgebungsverfahren. Nach $₫ 47 \mathrm{Abs} .3$ der im Jahre 2000 neu gefassten GGO soll der Entwurf einer Gesetzesvorlage den auf Bundesebene bestehenden Zentral- und Gesamtverbänden sowie den Fachkreisen möglichst frühzeitig zugeleitet werden, wenn ihre Belange berührt sind. Zeitpunkt, Umfang und Auswahl bleiben dabei ausdrücklich dem Ermessen des federführenden Bundesministeriums überlassen.

Tatsächlich erfolgt eine Einbindung der beteiligten Fachkreise und Verbände aber schon früher. Abgesehen von dem informalen und deshalb zwangsläufig nicht normierten Informationsaustausch sieht $₫ 44$ Abs. 5 Nr. 2 S. 2 GGO vor, dass das federführende Ministerium von den betroffenen Verbänden (und insbesondere auch der mittelständischen Wirtschaft) Angaben über die zu erwartenden Auswirkungen des Gesetzes auf Einzelpreise, das Preisniveau sowie auf die Verbraucher einholt. Im Vordergrund dieser Beteiligungsform steht also vor allem das Informationsinteresse der gesetzesvorbereitenden Ministerialverwaltung. Hintergrund ist, dass die Gesetzesentwürfe seit der neuen Geschäftsordnung auch zu einer Gesetzesfolgenabschätzung verpflichtet sind, die vor allem auf die finanziellen Auswirkungen auf die öffentlichen Haushalte in Bund, Ländern und Kommunen, daneben aber eben auch die Kosten für die Wirtschaft und die Auswirkungen auf die Verbraucher (sowie auf das Geschlechterverhältnis - $\$ 2$ GGO) gerichtet ist. ${ }^{60}$

Die Tatsache, dass den Geschäftsordnungen nach ganz überwiegender Meinung keine Außenverbindlichkeit zukommt, ihnen somit keine subjektiven Rechte der Betroffenen auf Beteiligung zu entnehmen sind und etwaige Verstöße auch grundsätzlich nicht zur Ungültigkeit der auf ihnen beruhenden Entscheidungen führen, ${ }^{61}$ wird im Schrifttum von einigen als Vorteil, ${ }^{62}$ von anderen als Nachteil empfunden. ${ }^{63}$

\section{Zulässigkeit eines Gesetzgebungsverfahrensrechts}

Hintergrund dieses Streits ist die Frage, ob dem Gesetzgeber - abgesehen von den ausdrücklich verfassungsrechtlich normierten Vorgaben - überhaupt irgendwelche Verfahrensvorgaben auferlegt werden dürfen, ob das innere Gesetzgebungsverfahren also einer (verbindlichen) rechtlichen Normierung zugänglich ist oder nicht.

61 $\$ 44$ Abs. 3 bis 6 GGO; näher Carl Böhret / Götz Konzendorf, Handbuch Gesetzesfolgenabschätzung (GFA), 2001, passim, sowie rechtsvergleichend jüngst Sylvia Veit, Bessere Gesetze durch Folgenabschätzung? Deutschland und Schweden im Vergleich, 2010, S. $52 \mathrm{ff}$.

${ }^{61}$ BVerfGE 1, 144 ff.; Klaus Stern, Das Staatsrecht der Bundesrepublik Deutschland, Bd. 2, Staatsorgane, Staatsfunktionen, Finanz- und Haushaltsverfassung, Notstandsverfassung, 1980, S. 307.

${ }^{62}$ Hans H. Klein, in: Maunz/Dürig (Hrsg.), Grundgesetz Kommentar, 64. EL 2012, Art. 40 Rn. 55 ff. m.w. N.

${ }^{63}$ Für eine weitergehende Beachtlichkeit Volker Haug, Bindungsprobleme und Rechtsnatur parlamentarischer Geschäftsordnungen, 1994, S. 141 f. 


\section{a) Meinungsstand}

Hier stehen sich zwei Ansätze - wie es scheint, unversöhnlich - gegenüber. Die eine findet sich in dem viel zitierten Satz von Klaus Schlaich wieder. Danach ,schuldet der Gesetzgeber ... gar nichts anderes als das Gesetz."64 Zuvor schon hat Willi Geiger ausgeführt: „Er schuldet weder eine Begründung noch gar die Darlegung aller seiner Motive, seiner Erwägungen und Abwägungen." ${ }^{65}$ Mit der Bindung des Gesetzgebers an die verfassungsmäßige Ordnung (Art. 20 Abs. 3 GG) und insbesondere an die Grundrechte (Art. 1 Abs. 3 GG) lässt sich dieser Satz in seiner Stringenz freilich nicht aufrechterhalten. So hat Klaus Schlaich korrigierend hinzugefügt: Der Gesetzgeber schuldet „ein wirksames, also gültiges und verfassungsmäßiges Gesetz. “66 Mit diesem Zugeständnis ist aber gleichsam eine Einbruchstelle auch für verfahrensbezogene Vorgaben geöffnet, wenn sie sich nur aus der Verfassung ableiten lassen.

Diesen Weg beschreitet die andere Position. Sie versucht, aus den verschiedensten Verfassungsbestimmungen das „Optimale Gesetzgebungsverfahren ${ }^{\text {“6 }} \hat{\imath}$ oder die „Pflicht zum guten Gesetz"68 als Verfassungspflicht abzuleiten. Namentlich Jörg Lücke schlägt vor, die aus der Verfassung abgeleiteten materiellen und formellen Grundpflichten in einer allgemeinen Gesetzgebungsordnung zu verankern, die, obgleich vom Gesetzgeber selbst erlassen, für eben diesen verbindlich sein soll. ${ }^{69}$ Bereits zuvor sind - unter anderem auch von Michael Kloepfer - Überlegungen zu einem Normenerlassrecht oder zu einem Gesetzgebungsverfahrensrecht angestellt worden. ${ }^{70}$ In gewisser Weise erinnern diese Konstruktionen an die Grundsatzgesetze oder das vom Bundesverfassungsgericht erfundene Maßstäbegesetz, ${ }^{71}$ wobei diese ihre sachliche Rechtfertigung freilich daraus beziehen, dass sie für Bund und Länder gleichermaBen verbindlich sein wollen.

Vermittelnd lässt sich zwar eine verfassungsrechtliche Pflicht zur optimalen Gesetzgebung ablehnen, weil nicht die abstrakte inhaltliche Richtigkeit, sondern ,die politische Akzeptanz unter Wahrung grundrechtlich geschützter Rechtspositionen und sonstiger verfassungsrechtlicher Vorgaben" die praktische Grundlage eines im demokratischen Prozess zustande gekommenen Gesetzes darstelle. ${ }^{72}$ Mit dieser $\mathrm{Ne}-$

${ }^{64}$ Klaus Schlaich, Die Verfassungsgerichtsbarkeit im Gefüge der Staatsfunktionen, VVDStRL 39 (1981), S. 99 (109).

${ }^{65}$ Willi Geiger, Gegenwartsprobleme der Verfassungsgerichtsbarkeit aus deutscher Sicht, in: Thomas Berberich u.a. (Hrsg.), Neue Entwicklungen im öffentlichen Recht, 1979, S. 141.

${ }^{66}$ Klaus Schlaich, in: ders. / Stefan Korioth, Das Bundesverfassungsgericht. Stellung, Verfahren, Entscheidungen, 8. Aufl. 2010, Rn. 542.

${ }^{67}$ Gunther Schwerdtfeger, Optimale Methodik der Gesetzgebung als Verfassungsplicht, in: Rolf Stödter/Werner Thieme (Hrsg.), Hamburg, Deutschland, Europa. Beiträge zum deutschen und europäischen Verfassungs-, Verwaltungs- und Wirtschaftsrecht, Festschrift für Hans Peter Ipsen zum 70. Geburtstag, 1977, S. $173 \mathrm{ff}$.

68 Axel Burghart, Die Pflicht zum guten Gesetz, Berlin 1996, passim.

${ }^{69} \mathrm{Jörg} \mathrm{Lücke,} \mathrm{Die} \mathrm{Allgemeine} \mathrm{Gesetzgebungsordnung,} \mathrm{ZG} \mathrm{2001,} \mathrm{S.} 1$ (43).

70 Michael Kloepfer, Abwägungsregeln bei Satzungsgebung und Gesetzgebung, Über Regelungen für den Erlaß von Rechtsnormen, DVBl. 1995, S. $441 \mathrm{ff}$.

71 BVerfGE 101, $158 \mathrm{ff}$.

72 Horst Risse, Verfassungsrechtliche und politische Grenzen des Gesetzgebungsoutsourcing, in: Michael Kloepfer (Hrsg.), Gesetzgebungsoutsourcing, 2011, S. 109 (115); Fritz Ossenbühl, in: Josef Isensee / Paul Kirchhof (Hrsg.), HdBStR Bd. 3, 1. Auf. 1988, \$63 Rn. 7. 
gierung einer verfassungsrechtlichen Pflicht muss nicht zugleich ausgeschlossen sein, dass aus dem Verfassungsrecht Vorgaben für die legislatorische Methode zur Regelung eines bestimmten Problemkreises erwachsen können, wenn dies auch weit hinter dem Konzept einer optimalen Gesetzgebung zurückbleibt. ${ }^{73}$

\section{b) Verfahrensvorgaben für sonstige abstrakt-generelle Regelungen}

Blendet man die demokratietheoretischen Überlegungen, die jedenfalls prinzipiell gegen eine über die Verfassung hinausgehende Bindung des parlamentarischen $\mathrm{Ge}$ setzgebers sprechen mögen, aus und versucht, aus der Perspektive des Verwaltungsrechts Funktionsbedingungen und -möglichkeiten des Verfassungsrechts zu entwickeln, ${ }^{74}$ so ist festzustellen, dass dem deutschen Rechtssystem der Gedanke, dass beim Erlass abstrakt-genereller Regelungen verbindliche Verfahrensvorgaben zu beachten sind, keinesfalls fremd ist.

So ist bspw. der Erlass von Satzungen zahlreichen formellen und materiellen Voraussetzungen unterworfen. Als Beispiel sei nicht nur auf die entsprechenden Vorgaben in den Gemeindeordnungen verwiesen, ${ }^{75}$ sondern insbesondere an den Erlass von Bebauungsplänen erinnert. ${ }^{76}$ Neben den umfangreichen und in diesem Kontext nicht weiter interessierenden Vorgaben für die planerische Abwägung sieht das Baurecht vielfältige Beteiligungen der betroffenen Bürger vor. ${ }^{77}$

Auch für den Erlass von Rechtsverordnungen ist mitunter eine Anhörung der Betroffenen vorgesehen. So bestimmen verschiedene Regelungsermächtigungen im BIMSchG, im KrW-/ AbfG, BbodSchG, im ChemG und im WRMG, dass beim Erlass bestimmter Rechtsverordnungen (wie im übrigen auch beim Erlass von Verwaltungsvorschriften und Zielfestlegungen) die „beteiligten Kreise“ anzuhören sind.

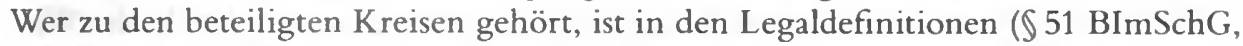
$\$ 60 \mathrm{KrW}-/ \mathrm{AbfG}, \S 20 \mathrm{BBodSchG}, \S 17 \mathrm{Abs} .7 \mathrm{ChemG}$, sowie $₫ 6$ WRMG) allerdings nur in allgemeiner Weise festgelegt. Die Betroffenen machen dabei nur eine zu beteiligende Gruppe neben denen der Wissenschaft, der beteiligten Wirtschaft sowie der jeweils zuständigen obersten Landesbehörden aus. Wer im Einzelnen anzuhören ist und wie das Verfahren im Einzelnen ausgestaltet ist, geht aus den Vorschriften über die Anhörung beteiligter Kreise nicht deutlich hervor. Unklar bleibt auch, welche Folgen sich aus dem Unterlassen der Anhörung oder aus sonstigen Verfahrensfehlern ergeben. Eine systematische Betrachtung der einzelnen Regelungsermächtigungen, die eine obligatorische Anhörung der beteiligten Kreise vorsehen, zeigt, dass die Anhörung beteiligter Kreise vor allem in solchen Fällen verlangt wird, in denen es darum geht, technisch-wissenschaftliche komplexe und interessenrelevante

${ }^{73}$ Horst Risse, Verfassungsrechtliche und politische Grenzen des Gesetzgebungsoutsourcing, in: Michael Kloepfer (Hrsg.), Gesetzgebungsoutsourcing, 2011, S. 109 (117); Fritz Ossenbühl, in: Josef Isensee/ Paul Kirchhof (Hrsg.), HdBStR Bd. 3, 1. Aufl. 1988, §63 Rn. 7.

${ }^{74}$ Instruktiv Jens Kersten, Was kann das Verfassungsrecht vom Verwaltungsrecht lernen?, DBVl 2011, S. $585 \mathrm{ff}$.

${ }^{75}$ Vgl. bspw. Art. 24 GO Bayern, $\$ 24$ GO Rheinland-Pfalz, $\$ \$ 6-8$ GO Sachsen-Anhalt.

76 Vgl. $\$ \$ 8$ ff. BauGB.

77 Grundlegend Ulrich Battis, Partizipation im Städtebaurecht, 1976, passim. 
Sachverhalte zu beurteilen. Nicht vorgeschrieben ist die Anhörung beteiligter Kreise dagegen von Regelungsermächtigungen, die dem Normgeber nur einen geringen eigenen Regelungsspielraum belassen, etwa weil sie der Umsetzung internationaler Standards dienen. ${ }^{78}$ Diese kurze Analyse zeigt, dass auch hier die Information des Normgebers im Vordergrund der Beteiligung steht.

\section{c) Übertragbarkeit auf formelle Gesetze}

Diese funktionale Kongruenz - oder besser: diese teilweise funktionale Kongruenz - zwischen der obligatorischen Einbindung der beteiligten Kreise in den Erlass von Rechtsverordnungen und der Betroffenenbeteiligung im Gesetzgebungsverfahren lässt möglicherweise auf die Zulässigkeit von verbindlichen Verfahrensregelungen für den Gesetzgeber schließen. Ebenso wie der Verordnungsgeber in bestimmten Fällen die beteiligten Kreise anzuhören hat, könnte der Gesetzgeber zu einer Beteiligung der Betroffenen verpflichtet sein. Namentlich Michael Kloepfer hat für diesen Rückschluss von gesetzlichen Normierungen auf gesetzliche Verpflichtungen einen methodischen Weg bereitet. ${ }^{79}$ Politisch geht es dabei um das Lernen des Gesetzgebers von sich selbst, indem dieser aus seinen eigenen Produkten modifizierte Selbst-Bindungen ableitet. Zu einer Verpflichtung wird dieser Lernprozess freilich erst, wenn der Gesetzgeber - möglicherweise unwissend - Verfassungsprinzipien konkretisiert hat, an deren Inhalte er in modifizierter Form selbst gebunden ist. ${ }^{80} \mathrm{Um}$ es ganz deutlich zu machen: Wenn es sich bei der obligatorischen Anhörung der beteiligten Kreise beim Erlass bestimmter Rechtsverordnungen um die gesetzliche Konkretisierung eines allgemeinen Verfassungsgebots handeln sollte, wäre der Gesetzgeber über Art. 20 Abs. 3 GG selbst an eben dieses Gebot gebunden.

Michael Kloepfer selbst hat diesen Ansatz bislang nicht weiter verfolgt. In der Wissenschaft ist er - soweit ersichtlich - eher auf Skepsis gestoßen. Zu unterschiedlich seien die Satzungsgebung und der Erlass von Rechtsverordnungen durch die Exekutive auf der einen Seite und die Gesetzgebung auf der anderen Seite. ${ }^{81}$ Beide Auffassungen sind dabei aber möglicherweise stets über das Ziel hinausgeschossen: Die Auffassung, nach der bestimmte Elemente des Verwaltungs-, insbesondere des Planungsrechts auf die Gesetzgebung angewendet werden könnten, hat stets versucht,

78 Vgl. bspw. $₫ \oint 7$ Abs. 4, 37, 39, 48a BImSchG; $₫ 65$ KrWG.

${ }^{79}$ Michael Kloepfer, Was kann die Gesetzgebung vom Planungs- und Verwaltungsrecht lernen? Zum Programm eines parlamentsbindenden Normenerlaßrechts, ZG 1988, S. 289 ff.; ders., Abwägungsregeln bei Satzungsgebung und Gesetzgebung, Über Regelungen für den Erlaß von Rechtsnormen, DVBl. 1995 , S. $441 \mathrm{ff}$.

${ }^{80}$ Hans-Joachim Konrad, Parlamentarische Autonomie und Verfassungsbindung im Gesetzgebungsverfahren, DÖV 1971, S.80; Gunther Schwerdtfeger, Optimale Methodik der Gesetzgebung als Verfassungspflicht, in: Rolf Stödter/Werner Thieme (Hrsg.), Hamburg, Deutschland, Europa. Beiträge zum deutschen und europäischen Verfassungs-, Verwaltungs- und Wirtschaftsrecht, Festschrift für Hans Peter Ipsen zum 70. Geburtstag, 1977, S. 173 ff; Michael Kloepfer, Was kann die Gesetzgebung vom Planungs- und Verwaltungsrecht lernen? Zum Programm eines parlamentsbindenden Normenerlaßrechts. ZG 1988, S. 289 (294).

${ }^{81}$ Alexander Windoffer, Verfahren der Folgenabschätzung als Instrument zur rechtlichen Sicherung von Nachhaltigkeit, 2011, S. $160 \mathrm{f}$. 
Parallelen zur Ermessens- und Abwägungsfehlerlehre zu entwickeln. ${ }^{82}$ An dieser massiven Beschränkung des gesetzgeberischen Prognose- und Gestaltungsspielraums mussten sich die Kritiker zu Recht stoßen. Das Gesetz sei als Ergebnis der demokratischen Entscheidung notwendig Ausdruck des Kompromisses sowie des politisch Gewollten, nicht aber der sachlichen Richtigkeit. ${ }^{83}$ Doch die grundsätzliche Absage an eine Übertragbarkeit verwaltungsverfahrensrechtlicher Institutionen auf die $\mathrm{Ge}$ setzgebung hat möglicherweise übersehen lassen, dass punktuell doch durchaus Parallelen geboten sind, die letztlich aus der gemeinsamen Verfassungsbindung von Exekutive und Legislative herrühren. ${ }^{84}$ Das gilt bspw. auch für die Frage der Anhörung von Betroffenen.

Das Thema der Anhörung war auch Gegenstand einiger Entscheidungen des Bundesverfassungsgerichts. Das erste Mal hatte sich das Bundesverfassungsgericht auf Beschwerde eines Schallplattenherstellers mit der Frage zu befassen, ob diese Firma im Gesetzgebungsverfahren, das auf die Änderung der Umsatzsteuer gerichtet war, hätte angehört werden müssen. ${ }^{85}$ Im Rahmen dieses Gesetzgebungsverfahrens sind eine ganze Reihe von Verbänden angehört worden, so dass sich die Beschwerde des Schallplattenherstellers insbesondere auf die Feststellung eines Gleichheitsverstoßes richtete. Das Bundesverfassungsgericht wies die Beschwerde indes mit der Begründung zurück, es läge grundsätzlich im Ermessen der Gesetzgebungsorgane und ihrer Ausschüsse, ob und welche Verbände und Sachverständige in einem Anhörungsverfahren zu Wort kommen sollten, das nicht von der Verfassung vorgeschrieben sei ${ }^{86}$

In einer weiteren Entscheidung (zum Verbot der Nachnahmesendung von Tieren) rügte das Bundesverfassungsgericht hingegen die Tatsache, dass eine gesetzliche $\mathrm{Re}-$ gelung ohne Anhörung der von dieser Regelung betroffenen Berufsgruppen in das Gesetz eingefügt wurde. Konkrete Folgen leitete es aus der fehlenden Anhörung aber noch nicht ab. ${ }^{87}$

Erst in späteren (und vereinzelten Fällen), die sich aber nur bedingt verallgemeinern lassen, hat das Bundesverfassungsgericht das Recht der Betroffenen auf Anhörung im Gesetzgebungsverfahren betont. Das betrifft etwa gesetzliche Eingriffe in die kommunale Selbstverwaltungsgarantie, die nach Auffassung des Bundesverfassungsgerichts eine entsprechende Anhörung voraussetzt. ${ }^{88}$ Des Weiteren hat das Bundesverfassungsgericht in der Entscheidung zur Südumfahrung Stendal - nicht zuletzt wegen der enteignungsrechtlichen Vorwirkung des Planungsgesetztes - be-

${ }^{82}$ Michael Kloepfer, Was kann die Gesetzgebung vom Planungs- und Verwaltungsrecht lernen? Zum Programm eines parlamentsbindenden Normenerlaßrechts, ZG 1988, S. 289 (301); ders., Abwägungsregeln bei Satzungsgebung und Gesetzgebung, Über Regelungen für den Erlaß von Rechtsnormen. DVBI. 1995, S. 441 (445).

${ }^{83}$ So schon vorher Christoph Gusy, I)as Grundgesetz als normative Gesetzgebungslehre? ZRP 1985, S. 291 (298f.).

${ }^{84}$ Gunther Schwerdifeger, Optimale Methodik der Gesetzgebung als Verfassungspflicht, in: Rolf Stödter/Werner Thieme (I Irsg.), Hamburg, Deutschland, Europa. Beitrage zum deutschen und europäischen Verfassungs-, Verwaltungs- und Wirtschaftsrecht, Festschrift für Hans Peter Ipsen zum 70. Geburtstag, 1977, S. 173 (178ff.).

${ }^{85}$ BVerfGE 36, $321 \mathrm{ff}$.

${ }^{B 6}$ BVerfGE 36, 321 (330 ff.).

87 BVerfGE 36, 47.

${ }^{88}$ BVerfGE 50, 195 (202); 86, 90 (107). 
sonderen Wert auf Anhörungs- und Beteiligungsrechte der Betroffenen gelegt. ${ }^{\text {yy }}$ Mögen diese Fälle nur kasuistisch zu fassen sein, so lässt sich doch schon erkennen, dass die Verpflichtung des Gesetzgebers, Betroffene im Gesetzgebungsverfahren frühzeitig anzuhören, dem Verfassungsrecht nicht so fremd ist, wie es die Gegner von Gesetzesverfahrensvorschriften gerne postulieren.

Hinzu kommt, dass in den genannten Entscheidungen letztlich der Rechtsschutzgedanke im Vordergrund des festgestellten bzw. betonten Rechts auf Anhörung stand, während sich eine allgemeine Pflicht des Gesetzgebers zur Anhörung von Betroffenen auch auf eine andere dogmatische Begründung stützen lässt.

In verschiedenen Entscheidungen begründete das Bundesverfassungsgericht nämlich die Pflicht des Gesetzgebers, sich vor Erlass eines Gesetzes über die bestehende Ausgangslage in korrekter und ausreichender Weise Kenntnis zu verschaffen. ${ }^{90}$ In der Literatur wird diese Pflicht zur zutreffenden und vollständigen Erfassung des Sachverhalts zu einer formellen Grundpflicht des Gesetzgebers erhoben.' Dogmatisch lässt sich dicsc Pflicht unter anderem mit dem Verhältnismäßigkeitsprinzip begründen, nach dem auch der Gesetzgeber verpflichtet ist, nur solche Mittel zu verwenden. die für die Erreichung des angestrebten Zweckes geeignet, erforderlich und angemessen sind. ${ }^{92}$ Aus dieser materiellen, auf den gesetzlichen Inhalt bezogenen Verpflichtung erwachsen der Legislative und - auf das Gesetzgebungsverfahren vorwirkend - den Gesetzesinitianten formelle Verpflichtungen. So verlangt das Prinzip der Erforderlichkeit, dass der Gesetzgeber prüfen muss, ob nicht aus tatsächlichen Gründen ein milderes aber gleich geeignetes Mittel vorzuziehen ist. ${ }^{93}$ Aus dieser grundsätzlichen Pflicht des Gesetzgebers zur Tatsachenermittlung folgt mittelbar auch die Sekundärpflicht, Sachverständige oder eben auch Betroffene - und sei es in Form von Verbandsvertretern - im Gesetzgebungsverfahren anzuhören.

\section{Ausgestaltung der Normierung}

Wenn eine Verpflichtung des Gesetzgebers zur Anhörung von Betroffenen also grundsätzlich zulässig erscheint, so könnte die Betroffenenbeteiligung in einem für den Gesetzgeber verbindlichen Rechtsakt näher konkretisiert werden. Konkretisierungsbedürftig sind insbesondere die Fragen, wann eine Anhörung stattzufinden hat, wer eine solche Anhörung durchzuführen hat, wie die Anhörung durchzuführen ist sowie vor allem die entscheidende Frage, wer überhaupt zum Kreis der Anzuhö-

89 BVerfGE 95, 1 (23).

90 Z.B. BVerfGE 39, 210 (226); 57, 139 (159f.); 65, 1 (55).

${ }^{91}$ Axel Burghart, Die Pflicht zum guten Gesetz, 1996, S. 124; Helmuth Schulze-Fielitz, Theorie und Praxis parlamentarischer Gesetzgebung - besonders des 9. Deutschen Bundestages (1980-1983), 1988, S. 490 .

92 Christoph Gusy, Das Grundgesetz als normative Gesetzgebungslehre?, ZRP 1985, S. 291 (295).

"33 Helmuth Schulze-Fielitz, Theorie und Praxis parlamentarischer Gesetzgebung - besonders des 9. Deutschen Bundestages (1980-1983), 1988, S. 385 f.; Gunnar Folke Schuppert, Verwaltungswissenschaft. Verwaltung, Verwaltungsrecht, Verwaltungslehre, 2000, S. 353. 
renden zählen soll, wer also Betroffener bzw. von einem Gesetz in besonderer Weise betroffen ist. ${ }^{94}$

Diese Frage scheint in vielen Fällen unmöglich zu beantworten. Denn zu unscharf werden sachliche, räumliche oder rechtliche Maßstäbe sein, um die besondere $\mathrm{Be}-$ troffenheit von der grundsätzlichen Betroffenheit abzugrenzen, die sich aus der Unterwerfung unter eine allgemeine generellere Norm nun einmal ergibt. Zusätzlich erschwert wird diese Abgrenzung, wenn man die zeitliche Komponente berücksichtigt und insoweit realisiert, dass sich der Kreis der Betroffenheit im Laufe der Geltung einer Norm verändern kann. Doch die Schwierigkeit, den Betroffenenkreis zu definieren, sollte nicht der maßgebliche Grund sein, eine Betroffenenbeteiligung im Gesetzgebungsverfahren nicht auf eine rechtliche Basis zu stellen. Denn erstens muss man sich darüber bewusst sein, dass wegen des abstrakt-generellen Charakters von Rechtsnormen stets eher ganze Personengruppen als einzelne Personen in besonderer Weise betroffen sein werden, so dass die Beteiligung der Betroffenen am Gesetzgebungsverfahren nur in den scltensten Fällen eine individuelle und eigene Einbindung in das Gesetzgebungsverfahren verlangt. Ausreichend ist vielmehr die repräsentative Beteiligung der betroffenen Personengruppe, bspw. durch einen Verbandsvertreter. Und zweitens und vor allem müssen weder absolute Kriterien für eine besondere Betroffenheit gefunden noch in relativer Weise definiert werden, welche Person oder Personengruppe von einer gesetzlichen Regelung im Vergleich zu dem allgemeinen Adressatenkreis in besonderer Weise berührt wird. Vielmehr kann insoweit doch dem schweizerischen Vorbild gefolgt werden, das im Vernehmlassungsgesetz, welches die verfassungsrechtliche Pflicht zur Vernehmlassung konkretisiert, zwischen "geladenen" und anderen Beteiligten unterscheidet und hinsichtlich der anderen schlicht und einfach auf das individuelle Interesse abstellt. ${ }^{95}$ Betroffen ist, wer sich in besonderer Weise interessiert.

Lässt sich die Betroffenenbeteiligung also insgesamt durchaus rechtlich fassen, dürfen die Nachteile einer solchen Regulierung doch nicht verkannt werden. Das Gesetzgebungsverfahren ist in weiten Teilen ein politischer Prozess mit zahlreichen Akteuren, der sich nur schwer formalisieren lässt. ${ }^{96}$ Die Gefahren, dass durch die verbindlichen Regelungen über die Betroffenenbeteiligung die Konsensfindung erschwert wird, dass das Gesetzgebungsverfahren insgesamt verlängert wird und dass

\footnotetext{
94 Skeptisch und zuversichtlich zugleich Helmuth Schulze-Fielitz, Theorie und Praxis parlamentarischer Gesetzgebung - besonders des 9. Deutschen Bundestages (1980-1983), 1988, S. 510.

${ }^{55}$ Art. 4 des schweizerischen Vernehmlassungsgesetzes lautet:

Abs. 1 Jede Person und jede Organisation kann sich an einem Vernehmlassungsverfahren beteiligen und eine Stellungnahme ein reichen.

Abs. 2 Zur Stellungnahme eingeladen werden:

a. die Kantone;

b. die in der Bundesversammlung vertretenen politischen Parteien;

c. die gesamtschweizerischen Dachverbände der Gemeinden, Städte und Berggebiete;

d. die gesamtschweizerischen Dachverbände der Wirtschaft;

e. die weiteren, im Einzelfall interessierten Kreise.

Abs. 3 Die Bundeskanzlei führt die Liste der Vernehmlassungsadressaten nach Absatz 2 Buchstaben a-d.

96 Vgl. Ulrich Karpen, Gesetzgebungs-, Verwaltungs- und Rechtsprechungslehre. Beiträge zur Entwicklung einer Regelungstheorie, 1989, S. 41.
} 
das Bundesverfassungsgericht einen weiteren Ansatzpunkt für die Verwerfung von Gesetzen erhält, sind nicht von der Hand zu weisen. ${ }^{97}$ Darüber hinaus muss man sich darüber bewusst sein, dass sich mit einer Betroffenenbeteiligung im Gesetzgebungsverfahren auch die Rationalitätsanforderungen an das Produkt des Gesetzgebungsverfahrens, an das Gesetz, ändern. ${ }^{98}$

Andererseits ist noch ein $\mathrm{Mal}$ an die Nachteile zu erinnern, die eine nichtregulierte Betroffenenbeteiligung für die Gesetzgebung mit sich bringt. Je mehr die eigentliche Entscheidungsfindung im politischen Prozess auf das innere Gesetzgebungsverfahren verlagert wird und je einflussreicher hier die Betroffenen sein können, desto wichtiger ist es, die grundsätzlich erst im äußeren Gesetzgebungsverfahren gewährleistete Öffentlichkeit auf den inneren Gesetzgebungsprozess zu erstrecken und für eine gleichmäßige und gleichberechtigte Beteiligung der verschiedenen Interessengruppen Sorge zu tragen.

Insofern wäre in einer Normierung der Betroffenenbeteiligung in jedem Fall vorzusehen, dass die Anhörung der Betroffenen öffentlich stattzufinden hat, soweit das mit ihren grundrechtlich geschützten Geheimnissen zu vereinbaren ist, dass die Ergebnisse der Anhörung protokolliert werden und dass die Begründung des letztlich zustande gekommenen Gesetzes darzulegen hat, inwieweit die Anhörung sich im Gesetzesinhalt widerspiegelt. Die Auswahl der anzuhörenden Verbände und Betroffenen muss nach kontrollierbaren Kriterien erfolgen und die Chancengleich heit gewährleisten. ${ }^{9 y}$ Nur wenn eine Betroffenenbeteiligung solchen Anforderungen genügt, wird sie auch die Vorteile hervorbringen können, die ihr zugedacht sind, wird sie vielleicht auch zu besseren Gesetzen beitragen können.

\section{Resümee}

Eine Betroffenenbeteiligung im Gesetzgebungsverfahren scheint ein geeignetes Instrument zu sein, um die parlamentarische Demokratie für partizipative bzw. direktdemokratische Aspekte behutsam zu öffnen. Doch ihre Vorteile wird sie nur entfalten können, wenn sie in rechtsverbindlicher Art und Weise normiert wird. Diese zusätzliche Regulierung des Gesetzgebungsverfahrens mittels eines Rechtsetzungsrechts ist nicht generell unzulässig, mag aber durchaus als nachteilig empfunden werden. Insoweit gibt es hinsichtlich einer Betroffenenbeteiligung im Gesetzgebungsverfahren nur zwei Möglichkeiten: Regulierung oder Verzicht.

97 Klaus Schlaich, Die Verfassungsgerichtsbarkeit im Gefüge der Staatsfunktionen, VVDStRL 39 (1981), S. 99 (115 ff.); Christoph Gusy, Das Grundgesetz als normative Gesetzgebungslehre?, ZRP 1985, S. 291 (298).

98 Instruktiv Helmuth Schulze-Fielitz, Theorie und Praxis parlamentarischer Gesetzgebung - besonders des 9. Deutschen Bundestages (1980-1983), 1988, S. $454 \mathrm{ff}$.

Hans-Joachim Mengel, Grundvoraussetzungen demokratischer Gesetzgebung, ZRP 1984, S. 153 (156). 